Supporting Information:

\title{
Competitive Adsorption as a Route to Area-Selective Deposition
}

\author{
Taewon Suh ${ }^{1}$, Yan Yang ${ }^{2}$, Pengyuan Zhao, ${ }^{1} \mathrm{Ka} \mathrm{Un} \mathrm{Lao}^{2}$, Hsin-Yu Ko ${ }^{2}$, Jonathan Wong ${ }^{2}$, \\ Robert A. DiStasio Jr. ${ }^{2 *}$, and James R. Engstrom ${ }^{1 *}$ \\ ${ }^{1}$ Robert F. Smith School of Chemical and Biomolecular Engineering \\ ${ }^{2}$ Department of Chemistry and Chemical Biology \\ Cornell University \\ Ithaca, NY 14853 USA
}

December 2019

Revised January 2020

ACS Applied Materials \& Interfaces manuscript am-2019-22065z

*Address correspondence to these authors: jre7@ cornell.edu or distasio@ cornell.edu 


\section{Binding Energetics.}

In this work, electronic binding energies $\left(\Delta U^{\mathrm{DFT}}\right)$ were computed via

$$
\Delta U^{\mathrm{DFT}}=U_{\mathbf{S}+\mathbf{A}}^{\mathrm{DFT}}-U_{\mathbf{S}}^{\mathrm{DFT}}-U_{\mathbf{A}}^{\mathrm{DFT}}
$$

in which $U_{\mathbf{S}+\mathbf{A}}^{\mathrm{DFT}}, U_{\mathbf{S}}^{\mathrm{DFT}}$, and $U_{\mathbf{A}}^{\mathrm{DFT}}$ are the total electronic (DFT) energies for the substrate + adsorbate $(\mathbf{S}+\mathbf{A})$, substrate $(\mathbf{S})$, and isolated adsorbate (A) systems. Finite temperature corrections to the internal binding energies $[\Delta U(T)]$ were evaluated at a given temperature $T$ as follows:

$$
\Delta U(T)=U_{\mathbf{S}+\mathbf{A}}(T)-U_{\mathbf{S}}(T)-U_{\mathbf{A}}(T),
$$

in which $U_{\mathbf{S}+\mathbf{A}}(T), U_{\mathbf{S}}(T)$, and $U_{\mathbf{A}}(T)$ are the total internal energies for the $\mathbf{S}+\mathbf{A}, \mathbf{S}$, and $\mathbf{A}$ systems given by:

$$
\begin{gathered}
U_{\mathbf{S}+\mathbf{A}}(T)=U_{\mathbf{S}+\mathbf{A}}^{\mathrm{DFT}}+U_{\mathbf{S}+\mathbf{A}}^{\mathrm{vib}}(T) \\
U_{\mathbf{S}}(T)=U_{\mathbf{S}}^{\mathrm{DFT}}+U_{\mathbf{S}}^{\mathrm{vib}}(T) \\
U_{\mathbf{A}}(T)=U_{\mathbf{A}}^{\mathrm{DFT}}+U_{\mathbf{A}}^{\mathrm{trans}}(T)+U_{\mathbf{A}}^{\mathrm{rot}}(T)+U_{\mathbf{A}}^{\mathrm{vib}}(T) .
\end{gathered}
$$

In these expressions, $U^{\mathrm{vib}}(T)$ is the vibrational contribution to $U(T)$, which has been computed within the harmonic approximation, and depends on the (harmonic) vibrational frequencies $(\omega)$ of the system via

$$
U^{\mathrm{vib}}(T)=\sum_{\omega}\left[\frac{\hbar \omega}{2}+\frac{\hbar \omega}{\exp \left[\hbar \omega / k_{B} T\right]-1}\right]
$$

in which $k_{\mathrm{B}}$ is the Boltzmann constant and $\hbar$ is the reduced Planck constant. For the $\mathbf{S}+\mathbf{A}$ systems, the computed phonon spectra often contained one (or two) low-lying imaginary frequencies (i.e., $\omega \approx 10 i \mathrm{~cm}^{-1}$ ), which resulted from frustrated rotations of the surface-bound adsorbate. When computing $U^{\mathrm{vib}}(T)$ in Eq. [S3], the contribution from each of these modes 
was treated as $k_{B} T$ (i.e., the limit of Eq. [S6] as $\omega \rightarrow 0$ ), which is consistent with the equipartition theorem. We note in passing that the contributions from these modes were negligible when computing the binding energetics reported in this work. $U_{\mathbf{A}}^{\text {trans }}(T)$ and $U_{\mathbf{A}}^{\text {rot }}(T)$ are the translational and rotational contributions to $U(T)$, which were computed using the ideal gas (IG) and rigid rotor (RR) approximations for the vapor-phase A system only:

$$
\begin{gathered}
U_{\mathrm{A}}^{\mathrm{trans}}(T)=\frac{3}{2} N k_{B} T=\frac{3}{2} n R T \\
U_{\mathrm{A}}^{\mathrm{rot}}(T)=\frac{3}{2} N k_{B} T=\frac{3}{2} n R T,
\end{gathered}
$$

in which $R$ is the gas constant and $n$ is the number of moles (taken as one throughout this work).

Binding enthalpies $(\Delta H(T, p))$ at finite $T$ and pressure $(p)$ values were computed via

$$
\Delta H(T, p)=H_{\mathbf{S}+\mathbf{A}}(T, p)-H_{\mathbf{S}}(T, p)-H_{\mathbf{A}}(T, p),
$$

in which $H_{\mathbf{S}+\mathbf{A}}(T, p), H_{\mathbf{S}}(T, p)$, and $H_{\mathbf{A}}(T, p)$ are the enthalpies for the $\mathbf{S}+\mathbf{A}, \mathbf{S}$, and $\mathbf{A}$ systems given by:

$$
\begin{gathered}
H_{\mathbf{S}+\mathbf{A}}(T, p)=U_{\mathbf{S}+\mathbf{A}}(T)+p V \approx U_{\mathbf{S}+\mathbf{A}}(T) \\
H_{\mathbf{S}}(T, p)=U_{\mathbf{S}}(T)+p V \approx U_{\mathbf{S}}(T) \\
H_{\mathbf{A}}(T, p)=U_{\mathbf{A}}(T)+p V \approx U_{\mathbf{A}}(T)+n R T,
\end{gathered}
$$

in which the $p V$ terms were taken to be negligible for the condensed-phase $\mathbf{S + A}$ and $\mathbf{S}$ systems. For the vapor-phase A system, we employed the IG equation of state to set $p V=n R T$.

To further understand the thermodynamic stability of these systems, we also computed the corresponding binding Gibbs free energies $(\Delta G(T, p))$ via

$$
\Delta G(T, p)=G_{\mathbf{S}+\mathbf{A}}(T, p)-G_{\mathbf{S}}(T, p)-G_{\mathbf{A}}(T, p),
$$

in which $G_{\mathbf{S}+\mathbf{A}}(T, p), G_{\mathbf{S}}(T, p)$, and $G_{\mathbf{A}}(T, p)$ are the Gibbs free energies for the $\mathbf{S}+\mathbf{A}, \mathbf{S}$, and $\mathbf{A}$ systems given by: 


$$
\begin{gathered}
G_{\mathbf{S}+\mathbf{A}}(T, p)=H_{\mathbf{S}+\mathbf{A}}(T)-T S_{\mathbf{S}+\mathbf{A}}^{\mathrm{vib}}(T) \\
G_{\mathbf{S}}(T, p)=H_{\mathbf{S}}(T)-T S_{\mathbf{S}}^{\mathrm{vib}}(T) \\
G_{\mathbf{A}}(T, p)=H_{\mathbf{A}}(T)-T\left[S_{\mathbf{A}}^{\operatorname{trans}}(T, p)+S_{\mathbf{A}}^{\mathrm{rot}}(T)+S_{\mathbf{A}}^{\mathrm{vib}}(T)\right] .
\end{gathered}
$$

In these expressions, $S^{\operatorname{trans}}(T, p), S^{\mathrm{rot}}(T)$, and $S^{\mathrm{vib}}(T)$ are the translational, rotational, and vibrational contributions to the entropy of a given system. $S^{\mathrm{vib}}(T)$ was computed within the harmonic approximation, and takes on the following form:

$$
S^{\mathrm{vib}}(T)=\frac{1}{2 T} \sum_{\omega} \hbar \omega \operatorname{coth}\left[\hbar \omega / 2 k_{B} T\right]-k_{B} \sum_{\omega} \ln \left[2 \sinh \left[\hbar \omega / 2 k_{B} T\right]\right] .
$$

For the $\mathbf{S + A}$ systems, the contributions from modes with imaginary frequencies were treated as 0 (i.e., the limit of Eq. [S17] as $\omega \rightarrow 0$ ), which is consistent with the third law of thermodynamics. We again note that the contributions from these modes were negligible when computing the binding energetics herein. $S_{\mathbf{A}}^{\text {trans }}(T, p)$ and $S_{\mathbf{A}}^{\text {rot }}(T)$ were computed using the IG and RR approximations for the vapor-phase A system only, i.e.,

$$
\begin{gathered}
S_{A}^{\text {trans }}(T, p)=\frac{5}{2} n R+n R \ln \left[\left(\frac{2 \pi M k_{B} T}{\hbar^{2}}\right)^{3 / 2} \frac{R T}{N_{A} p}\right] \\
S_{A}^{\mathrm{rot}}(T)=\frac{3}{2} n R+n R \ln \left[\frac{\pi^{2}}{\sigma}\left(\frac{T^{3}}{\Theta_{\mathrm{rot}, 1} \Theta_{\mathrm{rot}, 2} \Theta_{\mathrm{rot}, 3}}\right)^{1 / 2}\right],
\end{gathered}
$$

in which $M$ is the molecular mass of $\mathbf{A}, N_{\mathrm{A}}$ is Avogadro's number, $\sigma$ is the rotational symmetry number (with $\sigma=1$ for 4-octyne), $\Theta_{\text {rot }, 1}, \Theta_{\text {rot }, 2}, \Theta_{\text {rot }, 3}$ are the rotational temperatures corresponding to the three principal moments of inertia of $\mathbf{A}$, and $p$ is the vapor pressure of $\mathbf{A}$. 


\section{XPS analysis: $C(1 s)$.}

As described in the main manuscript, after thin-film deposition we conducted in situ analysis using XPS without air-break, focusing on the elements present in the substrates, thin film, and those possibly introduced by the reactants and the co-adsorbate. It must be mentioned that following reaction, all substrates were cooled to room temperature, while an acceptable pressure was achieved in the surrounding chamber. Often a few to several hours were required to achieve this pressure before transfer to the UHV chamber for analysis from XPS.

In Fig. S-1, we plot the integrated intensity for the $\mathrm{C}(1 \mathrm{~s})$ feature for the eight reaction conditions considered in Fig. 6 of the main text. Again these eight experiments represent eight unique combinations of substrate temperature and partial pressure of 4-octyne, and four of the eight led to selective growth. As a consequence, we have put these results into two categories: those that led to selective growth and those that did not. As may be seen in Fig. S-1, we do not observe a correlation between achieving selectivity and the intensity of the $\mathrm{C}(1 \mathrm{~s})$ feature. We also observe relatively significant intensity on the $\mathrm{SiO}_{2}$ surface, where growth of $\mathrm{ZrO}_{2}$ was unimpeded, and binding of 4-octyne is expected to be weak. As such, we are led to conclude that the intensity of $\mathrm{C}(1 \mathrm{~s})$ is mostly a consequence of post-CVD adsorption at room temperature of residual hydrocarbons in the reaction chamber.

\section{$A F M$ and $\mathrm{SEM}$ of $\mathrm{ZrO}_{2}$ thin film on $\mathrm{SiO}_{2}$.}

In Fig. S-2, we present two cross-sectional SEM images of the same sample we considered in the main manuscript, $c f$. Fig. 5(c). In Fig. S-3, we present two AFM images illustrating the size of defect features that one can detect with AFM. 


\section{CVD growth on a patterned $\mathrm{Cu} \mid \mathrm{SiO}_{2}$ wafer.}

For an additional demonstration of the effect of the co-adsorbate on the growth of $\mathrm{ZrO}_{2}$ thin films, we also considered CVD on a patterned substrate. Here, we generated a substrate possessing a line-and-space pattern, where the lines consist of $50 \mu \mathrm{m}$-wide $\mathrm{Cu}$ thin films (100 $\mathrm{nm}$ thick) formed using liftoff techniques, and the spaces consist of $50 \mu$ m-wide $\mathrm{SiO}_{2}$ thin films (200 nm thick thermally grown $\mathrm{SiO}_{2}$ on top of a $\mathrm{Si}$ substrate). We did not attempt to formally hydroxylate the exposed $\mathrm{SiO}_{2}$ layers, hence the density of $-\mathrm{OH}(a)$ may differ from the substrates that were considered in the main manuscript involving $\mathrm{Si}$ substrates terminated by chemical oxide. Growth was conducted under conditions where selectivity was found for the coupon samples with layers of only $\mathrm{SiO}_{2}$ or $\mathrm{Cu}$ (i.e., $p_{\mathrm{A}}=0.36$ Torr and $T_{\mathrm{s}}=120{ }^{\circ} \mathrm{C}$ ). Following $\mathrm{CVD}$, we conducted XPS in situ and before air-break to determine the approximate amount of $\mathrm{ZrO}_{2}$ deposition.

In Fig. S-4, we present the results from ARXPS for the $\operatorname{Zr}(3 d)$ and $\operatorname{Si}(2 p)$ peaks. For the former, we have normalized the intensity to that observed at a takeoff angle $(\Theta)$ of $60^{\circ}$, while for the latter we plot the ratio of the intensity measured in situ after growth to that measured before growth (and after annealing to $180{ }^{\circ} \mathrm{C}$ ). For the $\mathrm{Cu}\left(2 \mathrm{p}_{3 / 2}\right)$ feature (not shown), we find no change in the intensities as a function of takeoff angle, suggesting no growth on top of $\mathrm{Cu}$. In Fig. S4(a), we see that the intensity of the $\operatorname{Zr}(3 \mathrm{~d})$ peak increases with increasing takeoff angle, consistent with the formation of a thin film on top of the substrate. For the $\operatorname{Si}(2 \mathrm{p})$ signal displayed in Fig. S-4(b), we observe significant attenuation with increasing takeoff angle, consistent with the formation of a thin film on top of those regions consisting of $\mathrm{SiO}_{2}$. We have also made use of the data shown in Fig. S-4 to make an estimate for the thin-film thickness. We 
assume that the $\mathrm{Si}(2 \mathrm{p})$ photoelectron intensity is attenuated by the overlying thin film of $\mathrm{ZrO}_{2}$ via

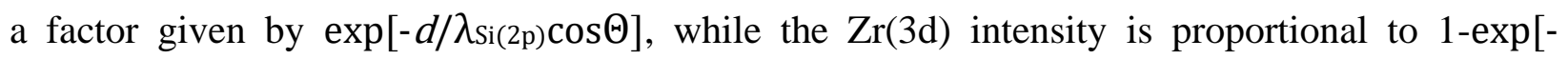

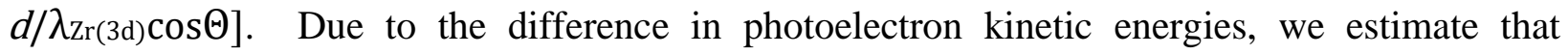
$\lambda_{\mathrm{Si}(2 \mathrm{p})} / \lambda_{\mathrm{Zr}(3 \mathrm{~d})}$ is $\sim 1.02$. The result of a fit to the two sets of data is shown by the solid lines in Fig. S-4, and we estimate that the thin-film thickness $d \sim 1.2 \mathrm{~nm}$.

We have also conducted a spatially resolved analysis conducted ex situ using scanning Auger on the sample "as-received" (i.e., no attempts we made to remove any surface contamination) due in part to our estimates for the thickness of the thin film deposited. The elemental maps produced from this technique have been spatially averaged along the direction of the line-and-space pattern to produce a 1D representation of the variation in composition across the line-and-space pattern. We plot these variations for $\mathrm{Cu}, \mathrm{Si}$, and $\mathrm{Zr}$ as a function of position in Fig. S-5. As can be seen, the line-and-space pattern of the underlying substrate is revealed with the expected variation of the $\mathrm{Cu}$ and $\mathrm{Si}$ signals. We also observe essentially perfect alignment of the $\mathrm{Zr}$ intensity with the $\mathrm{Si}$ signal, verifying the area-selective nature of the process we have developed herein. Due to signal-to-noise considerations, we cannot completely exclude the possibility that small amounts of $\mathrm{Zr}$ have been deposited in the $\mathrm{Cu}$ regions (about $75 \%$ of the pixels gave " 0 " for $\mathrm{Zr}$ in these regions).

\section{Acknowledgments}

We acknowledge the contributions of Chuck Hitzman of the Stanford Nano Shared Facility concerning the results from the scanning Auger microprobe. 

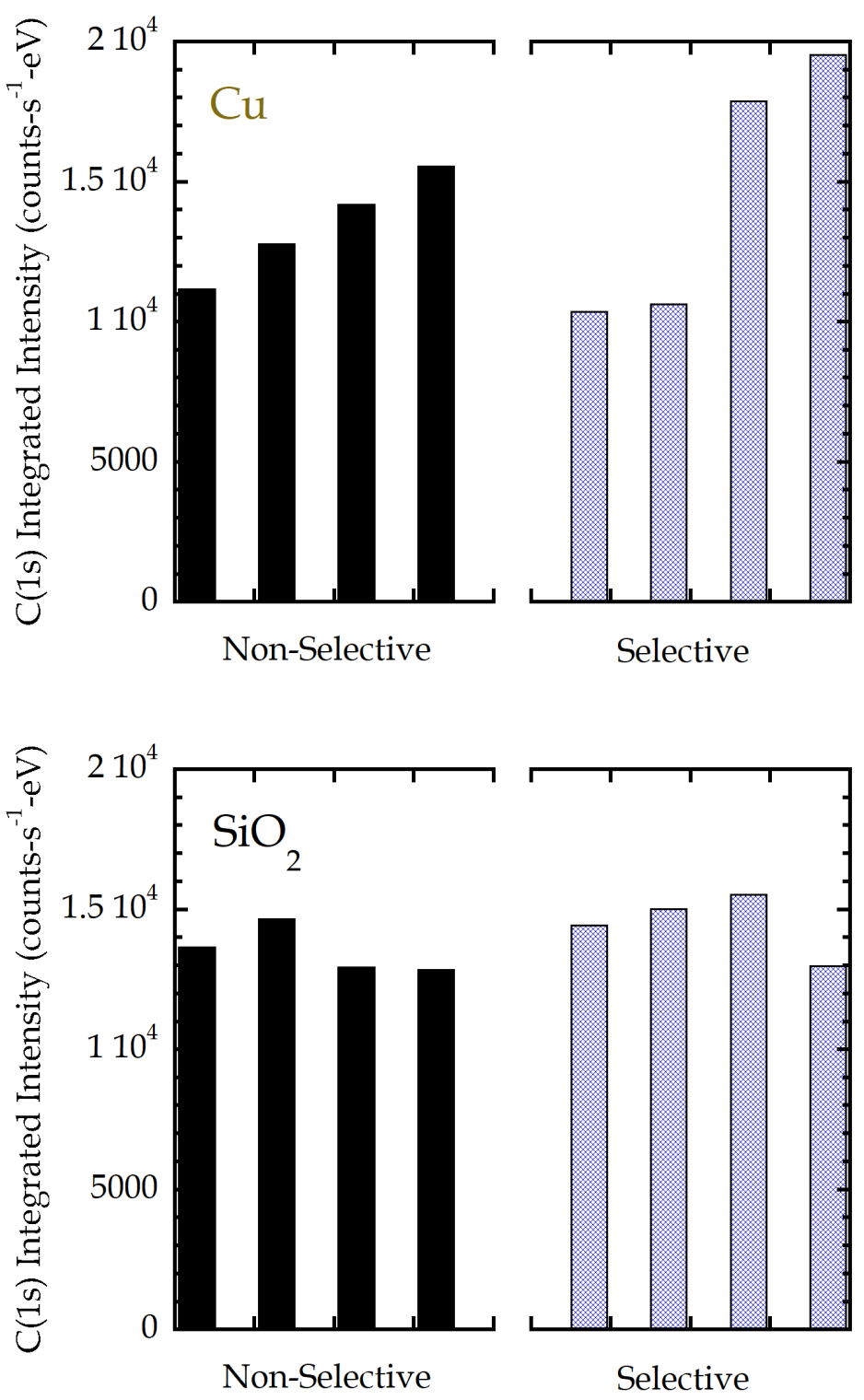

Figure S-1 Integrated intensities of the $\mathrm{C}(1 \mathrm{~s})$ peak for the experimental conditions considered in Fig. 6 of the main text. The data are shown for both surfaces $\left(\mathrm{Cu}\right.$ and $\left.\mathrm{SiO}_{2}\right)$ and have been grouped as to whether or not the reaction conditions gave selective growth. 

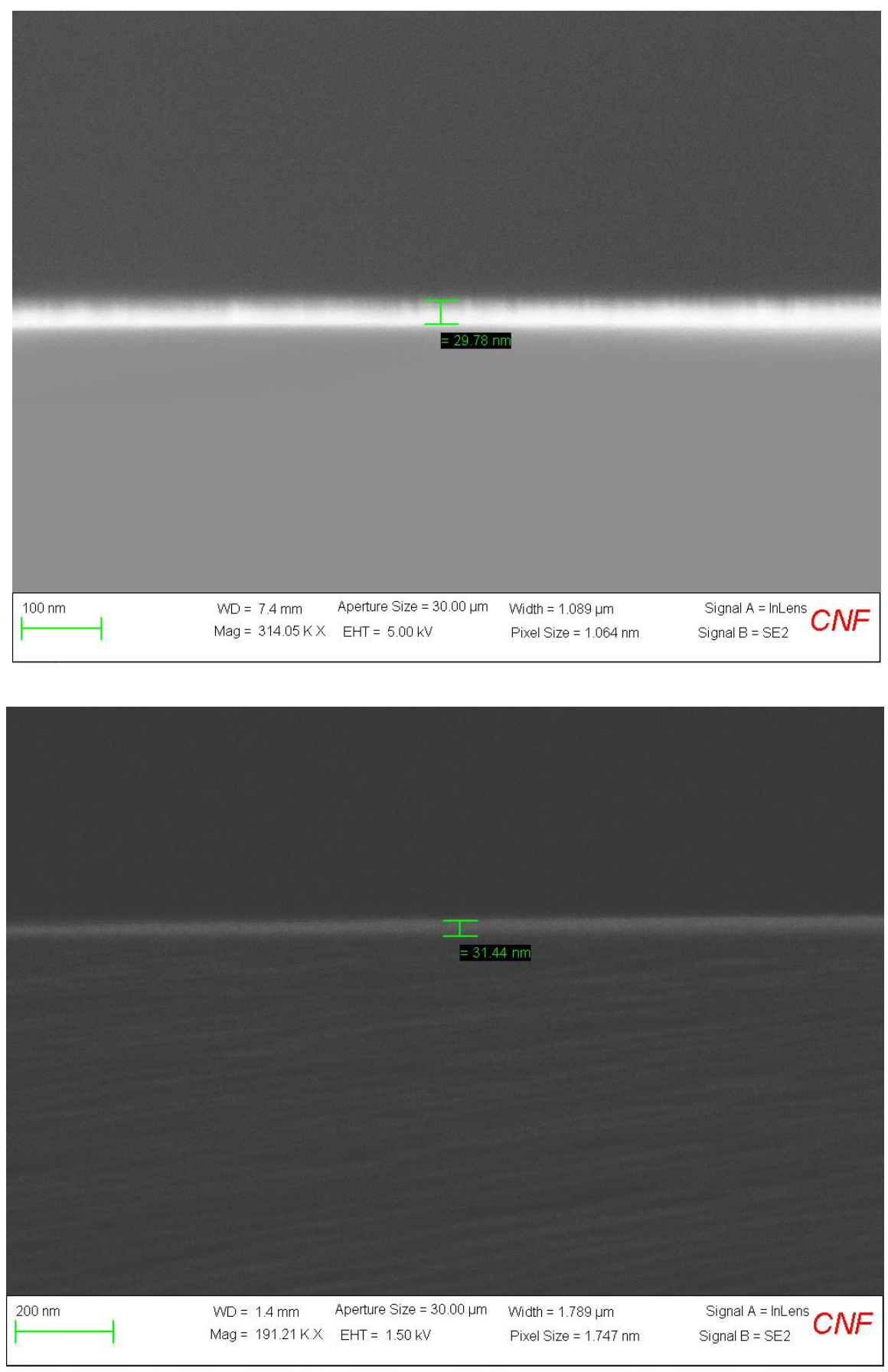

Figure S-2 Cross-sectional SEM images of the same sample that was considered in Fig. 5(c) of the main text. 

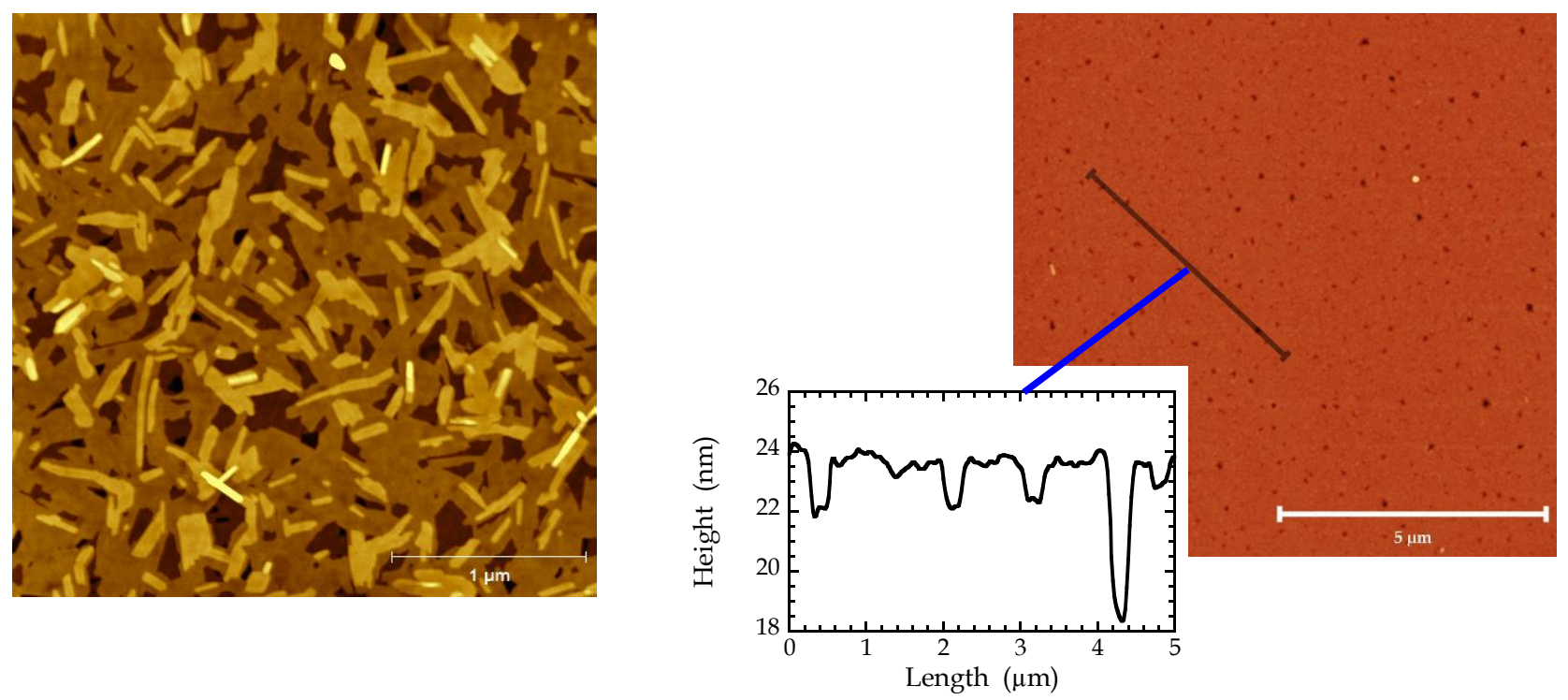

Figure S-3 Example AFM images of deposited thin films. Left panel: A $8 \mathrm{~nm}$ thick film of an organic semiconductor (PTCDI-C8), $3 \times 3 \mu \mathrm{m}^{2}$ scan (Ref. 1). Vacancy islands as small as $\sim 0.02 \mu \mathrm{m}$ can be imaged. Right panel: $\mathrm{An} \mathrm{ZrO}_{2}$ thin film $(\sim 3 \mathrm{~nm}$ thick) deposited by $\mathrm{ALD}$ on a $\mathrm{Cu}$ surface using a non-selective process, $10 \times 10$ $\mu \mathrm{m}^{2}$ scan. Pits (in the substrate and/or thin film) as small as $\sim 0.07 \mu \mathrm{m}$ can be imaged. 


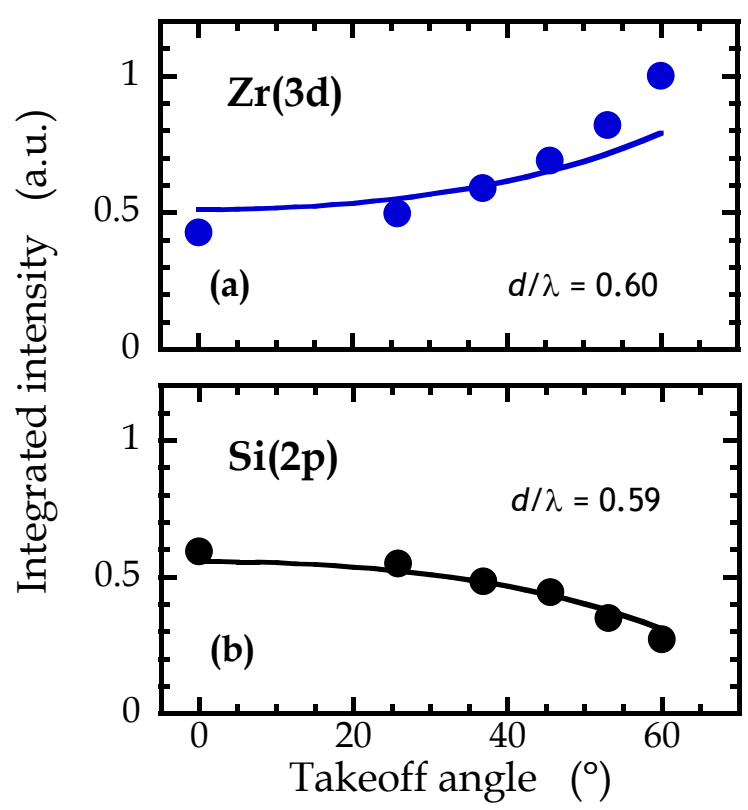

Figure S-4 Photoelectron intensities found from in situ angle-resolved XPS (ARXPS) of (a) the $\mathrm{Zr}(3 \mathrm{~d})$ peak and (b) the $\mathrm{Si}(2 \mathrm{p})$ peak for $\mathrm{ZrO}_{2} \mathrm{CVD}$ conducted on a wafer with a line-and-space pattern of $\mathrm{Cu}$ and $\mathrm{SiO}_{2}$. The partial pressure of the co-adsorbate (4-octyne) was $p_{\mathrm{A}}=0.36$ Torr and the substrate temperature was $T_{\mathrm{s}}=120{ }^{\circ} \mathrm{C}$. The solid lines represent a combined fit of both sets of data to a model that assumes that $\mathrm{ZrO}_{2}$ is deposited in the form of a uniform thin film only on those regions where $\mathrm{SiO}_{2}$ is present. 


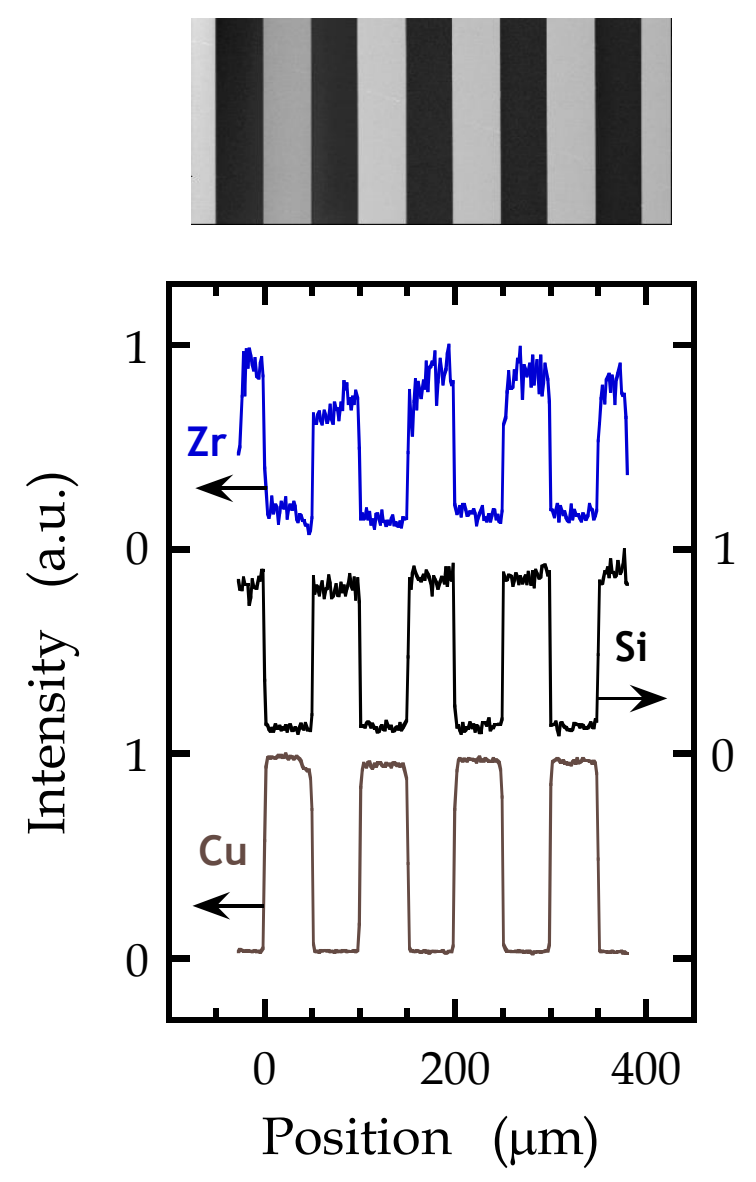

Figure S-5 Top: Plan view SEM of the patterned wafer with $50 \mu \mathrm{m} \mathrm{Cu}$ lines, spaced by 50 $\mu \mathrm{m} \mathrm{SiO}_{2}$ regions. Bottom: Normalized intensities obtained from ex situ scanning Auger microprobe analysis for $\mathrm{Zr}, \mathrm{Si}$ and $\mathrm{Cu}$ following $\mathrm{ZrO}_{2} \mathrm{CVD}$ conducted with 4-octyne as described in connection with Fig. S-4. The Auger intensities have been averaged along the direction of the $\mathrm{Cu}$ lines and normalized to a peak value of unity. 
Table S-1 Thin-film thickness values of $\mathrm{ZrO}_{2}$ on $\mathrm{SiO}_{2}$ (from ex situ $\mathrm{SE}$ ) and $\mathrm{Cu}$ (from in situ XPS) in Fig. 5(a) of the main text, in addition to the calculated selectivity values plotted in Fig. 5(b) of the main text.

\begin{tabular}{|c|c|c|c|}
\hline $\mathrm{O}_{2}$ Exposure (s) & Thickness (nm) on $\mathrm{SiO}_{2} / \mathrm{SE}$ & Thickness (nm) on Cu / XPS & Selectivity (\%) \\
\hline 2 & 3.69 & 0.009 & 99.5 \\
\hline 5 & 5.66 & 0.021 & 99.2 \\
\hline 10 & 8.31 & 0.013 & 99.7 \\
\hline 20 & 21.8 & 0.023 & 99.8 \\
\hline 30 & 28.8 & 0.065 & 99.6 \\
\hline
\end{tabular}




\section{References}

1. Kish, E. R., Nahm, R. K.; Woll, A. R.; and Engstrom, J. R. When the Sequence of Thin Film Deposition Matters: Examination of Organic-on-Organic Heterostructure Formation Using Molecular Beam Techniques and in Situ Real Time X-ray Synchrotron Radiation, J. Phys. Chem. C 2016, 120, 6165-6179. 